\title{
An Intelligent Driving and Management System of Solar LED with Adaptive Disturbance Observation Method
}

\author{
Qing $\mathrm{Hu}^{1, *}$, Xiaodan $\mathrm{Du}^{2}$ and Hui Chen ${ }^{3}$ \\ ${ }^{1}$ College of Information Science and Engineering, Chengdu University, Chengdu, China \\ 2 Information Network Center, Chengdu University, Chengdu, China \\ ${ }^{3}$ College of Electronic and Information Engineering, Southwest University of Nationalities, Chengdu, China \\ ${ }^{*}$ Corresponding author
}

\begin{abstract}
Solar power has become one of the most important renewable resources due to the energy crisis recently. An intelligent solar LED lighting driver system based on embedded technology was designed to make full use of the green energy. Electricity which was detected real-timely was generated by solar cells. Boost-Buck DC/DC converter was controlled by PWM to realize the control of Maximum Power Point Tracking Charge and the intelligent management to the batteries. Besides, LED was adopted to improve the energy efficiency, working reliability, practicality and environment protection. The new system is efficient, stable and practical. It can be widely applied in all kinds of embedded devices to supply the effective and environment protective energy.
\end{abstract}

Keywords-intelligent driving and management system; adaptive disturbance observation method; maximum power point tracking

\section{INTRODUCTION}

Solar energy is one of the focus of green energy currently. Among them, the photovoltaic battery power is mainly used. However, photovoltaic cells with obvious nonlinear output characteristics are vulnerable to the outside world and their own factors. A new type of solar LED lighting drive system based on embedded technology is designed to improve the overall efficiency of the photovoltaic power generation system and obtain the maximum power output. It can be widely used in the design of all kinds of embedded devices power supply.

\section{GENERAL STRUCTURE DESIGN OF THE SYSTEM}

Solar LED lighting driver hardware system based on embedded includes the controller module, solar batteries, DC/DC converter[1], Maximum Power Point Tracking (MPPT) charging control, batteries to store electrical energy, LED lighting driver controlled by PWM[2], the LED light source and the terminal management platform. The structure of the system is shown in Figure 1.

The way it works is, when sunlight exists, under the control of the embedded control chip, the solar battery will collect solar energy, convert it into electricity and store it by using the step-up step-down dual mode topology circuit structure, adopting the MPPT method and with the help of an intelligent charging management chip. When LED lighting system need electricity power supply, safe and efficient voltage and current is provided by using PWM control driving mode to make it safe, stable, efficient and reliable. There are two modes for adjusting the brightness of LED lighting source. One is intelligent light-adjusted mode which can adjust the brightness according to the strength of the external environment light, the other is artificial mode. The manmachine interactive interface of the terminal management platform can monitor temperature, voltage and current in real time for the system anomaly detection and maintenance and it provides clean environmental and safe green light for our work and life.

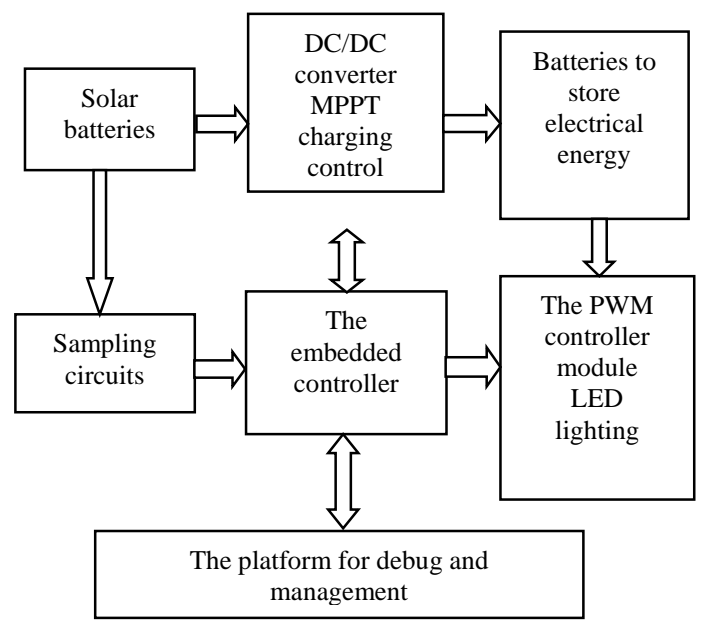

FIGURE I. FIGURE OF THE GENERAL STRUCTURE DESIGN

\section{SYSTEM SOFTWARE DESIGN}

Software design of the system includes the maximum power point tracking, current and voltage detection, temperature detection, light detection, intelligent management of the battery and LED lighting, human-computer interaction interface and terminal management platform. The key is the maximum power point tracking. 


\section{A. The Maximum Power Point tracking}

The maximum power point is tracked in the design by using PWM as the control parameter and the way of variable step, namely adaptive disturbance observation method [3][4]. A larger step is selected to close to the best working state of the system with a faster speed when far away from the maximum power point, and a smaller step is selected to close to the best working state of the system and reduce or avoid the oscillation of the system when close to the maximum power point. The Power-Duty ratio (P-D) relationship is shown in Figure 2.

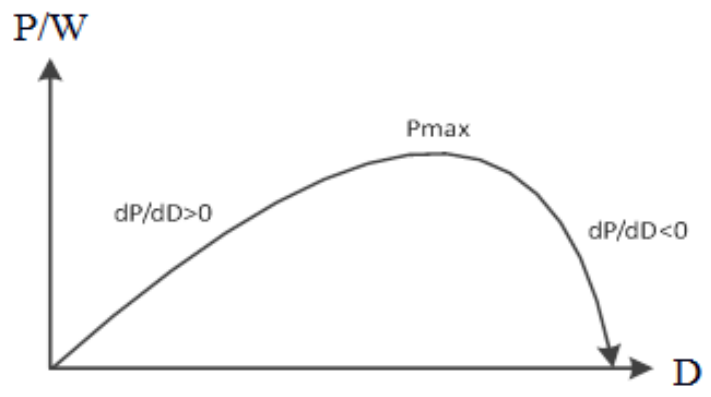

FIGURE II. FIGURE OF P-D RELATIONSHIP

\section{B. The Program Design of MPPT Algorithm with Adaptive}

\section{Disturbance Observation Method [5]}

The Figure 2 shows that $|d P / d D|$, the absolute value of the derivative of power duty ratio, is getting smaller when close to the maximum power point. The real-time step is constructed as follows:

$$
K+l=k p|d P| / K(l)
$$

Among it, $K+l$ is the adjusting step of Duty Cycle $(D)$, $0<K<1 ; d P=P(K)-P(K-l)$, is the change of the power; $k p$ is the scaling factor. (1) shows that the step $K+l$ is smaller when the power has smaller change to ensure the smoothness of the output power, and the step is larger when the power has bigger change to track the maximum power point quickly for making the system with some self-adaptive capacity. The scaling factor $\mathrm{kp}$ represents the size of the system sensitivity. The larger the more sensitive.

The program flow of adaptive disturbance observation method is shown in Figure 3 according to the above analysis [6] [7]. First, the current output power $P(K)$ is calculated by using the value of $U$ and $I$ to get $d P=P(K)-P(K-I)$. CMP which is the threshold set by the system determines control precision of the controller. When $|d P|$ is smaller than $C M P$, the system is considered working near the maximum power. No need to adjust the duty ratio $D$ at this point. When $|d P|$ is greater than $C M P$, it shows the current working point is far away from the maximum power point. The new step needs to be calculated to adjust the duty ratio $D$. Next, determine the symbol of $d P$. If it is positive, it continues to change in the original direction. If negative, it should change in the opposite direction. Variable flag is the sign bit of the step and it determines the change direction of the duty ratio $D$. The value of variable flag is -1 or 1 .

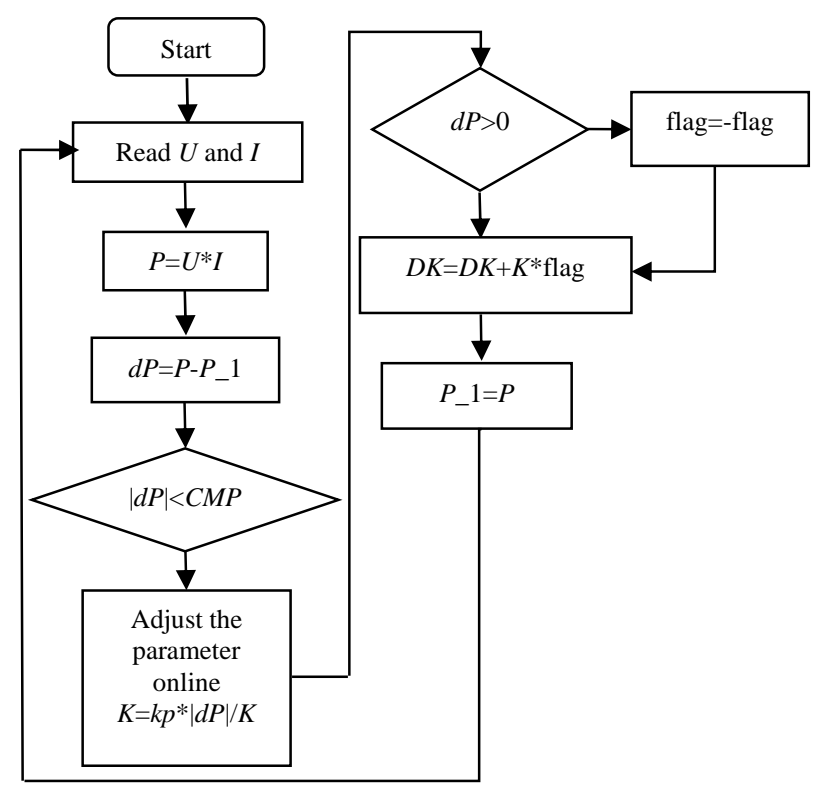

FIGURE III. FIGURE OF THE PROGRAM FLOW OF ADAPTIVE DISTURBANCE OBSERVATION METHOD

\section{The Management Platform}

The LCD panel debugging platform and mobile terminal management platform are designed to ease debugging and managing. While various parameters, such as the output voltage and power of the solar cell, the current of the charging loop, the temperature of components in the system, the capacity of the battery, the charging and discharging status, are debugged on the system board, they also are distributed and displayed on the terminal management platform by Bluetooth devices. Effective controls, such as sounding alarm and stopping work when a system exception (temperature) occurs, adjusting the brightness of LED light source artificially and stop charging, are presented in the system by the platform. It sets up a humanized human-computer interaction interface. The LCD panel debugging platform and mobile terminal management platform as shown in Figure 4 and Figure 5.

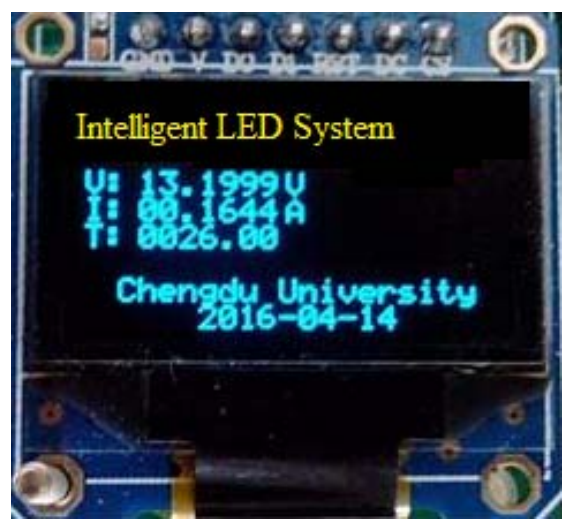

FIGURE IV. FIGURE OF THE LCD PANEL DEBUGGING PLATFORM 


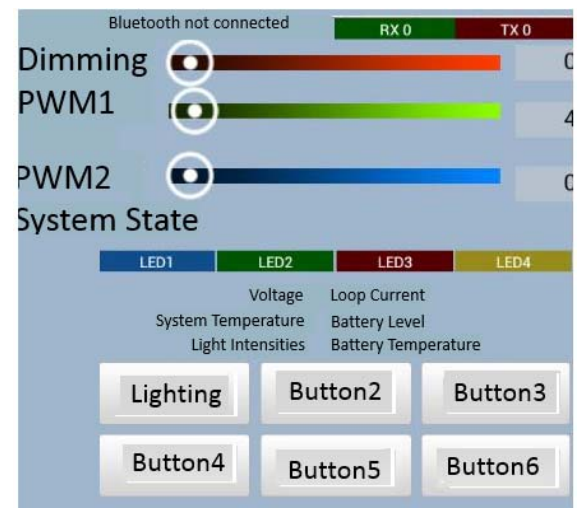

FIGURE V. FIGURE OF THE MOBILE TERMINAL MANAGEMENT PLATFORM

\section{EXPERIMENT RESULTS}

The solar panels whose open circuit terminal voltage is $21.5 \mathrm{~V}$, short-circuit current is $0.57 \mathrm{~A}$ and maximum power is $10 \mathrm{~W}$ are used in the experiment. All parameters can be observed and the brightness of the LED is controlled through the management platform. The charging loop current with MPPT and without MPPT are observed by the debugging platform in the case of the same output voltage in different time period. By comparison experiments of the power, it is easily observed that the efficiency of the photovoltaic power generation system has improved by MPPT. The experimental data is shown in Figure 6.

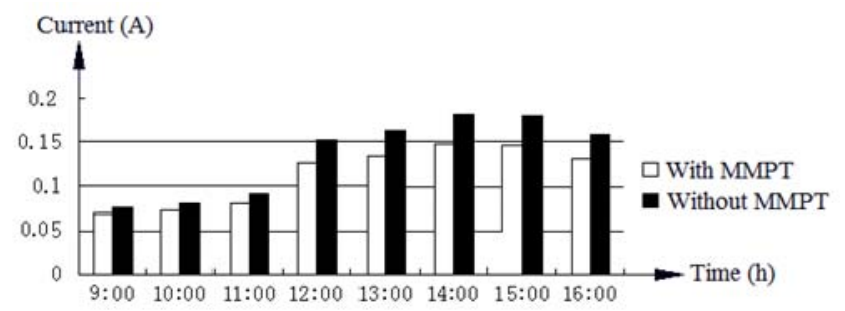

FIGURE VI. FIGURE OF THE EXPERIMENTAL DATA OF THE CHARGING LOOP CURRENT WITH MPPT AND WITHOUT MPPT

\section{CONCLUSIONS}

The solar LED lighting drive system is designed by using the embedded chip as the control circuit. It has simple structure, good reliability, high efficiency and various protections in various exceptions, such as the overcharge and the over discharge of the batteries, output overcurrent, etc. Based on the working principle of solar panel, according to the requirements for the energy control system, the maximum power point tracking control strategies are presented and improved. The real-time monitoring to the state of the various modules can be carried on. The brightness of LED light source can be adjusted according to the external environment intelligently, or according to the will of the individual artificially. In addition, USB power supply interface with 5V is as the extended design to plant to other devices easily. It has certain market value.

\section{ACKNOWLEDGMENT}

This project is supported by the nature and science fund from Sichuan Science and Technology Agency (2015GZ0275).

\section{REFERENCES}

[1] M. Fathi, A. Aissat and M. Abderrazak, "Optimization of the Electronic Driver and Thermal Management of LEDs Lighting Powered by Solar PV,” Energy Procedia, vol. 18, pp. 291-299, 2012.

[2] M. Fathi, A. Chikouche and M. Abderrazak, "Design and realization of LED driver for solar street lighting applications,” Energy Procedia, vol. 6, pp. 160-165, 2011.

[3] Lu Xiao, Qin Jun, "The application and simulation of adaptive disturbance observation method in MPPT of photovoltaic systems," Modern Electric, vol. 2, pp. 80-84, 2011.

[4] Wu Huabo, "The realization of the maximum power point tracking based on disturbance observation method," Electrical Measurement and Instrumentation, vol. 11, pp. 42-45, 2010.

[5] S. Saravanan, N. Ramesh Babu, "Maximum power point tracking algorithms for photovoltaic system - A review," Renewable and Sustainable Energy Reviews, vol. 57, pp. 192-204, 2016.

[6] Joe-Air Jiang, Tsong-Liang Huang, Ying-Tung Hsiao and Chia-Hong Chen, "Maximum Power Tracking for Photovoltaic Power System," Tamkang Journal of Science and Engineering, vol. 8, pp. 147-153, 2005.

[7] David Ouoba, Abderrahim Fakkar, Youssef El Kouari, Fayrouz Dkhichi and Benyounes Oukarfi, "An improved maximum power point tracking method for a photovoltaic system,” Optical Materials, vol. 56, pp. 100106, 2016. 